\title{
Vascular reactivity of arteria femoralis in adult and aged spontaneously hypertensive and Wistar-Kyoto rats
}

\author{
Petrova $\mathrm{M}^{1}$, Liskova $\mathrm{S}^{1,2}$, Vojtko $\mathrm{R}^{1}$, Villaris $\mathrm{R}^{1}$, Varga $\mathrm{Z}^{1}$, Zicha $\mathrm{J}^{2}$, Kristova $\mathrm{V}^{1}$ \\ Department of Pharmacology and Clinical Pharmacology, Faculty of Medicine, Comenius University, \\ Bratislava, Slovakia. miriam.petrova@fmed.uniba.sk
}

\begin{abstract}
Aim: The relationship of age and hypertension on endothelial dysfunction and increased responses to vasoconstrictor stimuli.

Background: Hypertension is a disease accompanied by endothelial dysfunction and is characterized by an impaired vascular reactivity and enhanced activity of sympathetic nervous system.

Materials and methods: In our experiment, we used spontaneously hypertensive rats representing model of essential hypertension and the Wistar-Kyoto rats as normotensive strain. Femoral arteries of adult and aged rats were put into the chamber of Mulvany-Halpern isometric myograph. As the nutrient solution, the modified Krebs-Henseleit solution having temperature $37^{\circ} \mathrm{C}$ and bubbled with $\mathrm{O}_{2}$ was used. After 30 minutes stabilization of blood vessels, a dose-dependent curve of norepinephrine response was recorded (concentrations $3 \times 10^{-8} \mathrm{M}$, $10^{-7} \mathrm{M}, 3 \times 10^{-7} \mathrm{M}, 10^{-6} \mathrm{M}, 3 \times 10^{-6} \mathrm{M}, 10^{-5} \mathrm{M}, 3 \times 10^{-5} \mathrm{M}, 10^{-4} \mathrm{M}$ ), followed by a dose-dependent curve of acetylcholine response (concentrations $3 \times 10^{-8} \mathrm{M}, 10^{-7} \mathrm{M}, 3 \times 10^{-7} \mathrm{M}, 10^{-6} \mathrm{M}, 3 \times 10^{-6} \mathrm{M}$ ).

Results: Our experiments recorded an increased reactivity to contraction stimuli in spontaneously hypertensive animals. Vascular reactivity to norepinephrine at 5 month and 12 month old rats from the same group was not significantly affected. Our experiments on the other hand, did not record a reduced endothelium-dependent relaxation in hypertensive compared to normotensive animals, neither in different age groups.

Conclusions: Increased norepinephrine-induced contraction occurs even before development of reduced acetylcholine-induced relaxation in SHR rats. We predict that in our experiment hypertension plays a bigger role in the development of endothelial dysfunction than aging (Fig. 2, Ref. 22). Full Text in PDF www.elis.sk. Key words: spontaneously hypertensive rats, Wistar-Kyoto rats, acetylcholine, norepinephrine.
\end{abstract}

Hypertension is disease, which is accompanied by impaired vascular reactivity and endothelial dysfunction, both in humans and in experimental models. To better understand the pathophysiology of an increased blood pressure, many animal models were developed either with induced or genetic form of hypertension. The most widely used include spontaneously hypertensive rats, stroke-prone hypertensive rats, chronic angiotensin II infusion, transgenic animals overexpressing the renin and the angiotensinogen gene, renovascular hypertension, Dahl salt-sensitive hypertension, deoxycorticosterone acetate-salt hypertension and endothelin-1 induced hypertension (Félétou and Vanhoutte, 2006). These models are similar to human forms of increased blood pressure and they can help better understand the mechanism of hypertension and the changes in reactivity of blood vessels to different stimuli.

${ }^{1}$ Department of Pharmacology and Clinical Pharmacology, Faculty of Medicine, Comenius University, Bratislava, Slovakia, and ${ }^{2}$ Center of Cardiovascular Research and Institute of Physiology, Academy of Sciences of the Czech Republic, Prague, Czech Republic

Address for correspondence: $\mathrm{M}$. Petrova, MD, PhD, Department of Pharmacology and Clinical Pharmacology, Faculty of Medicine, Comenius University, Sasinkova 4, SK-813 72 Bratislava, Slovakia. Phone: +421.911815119

Acknowledgement: The experimental work was supported by the research grants VEGA MŠ 1/0501/11.
According to animal models and the studied vascular bed, endothelial dysfunction can have different mechanisms. In our study, we used spontaneously hypertensive rats and compared them to normotensive Wistar-Kyoto rats. We selected spontaneously hypertensive rats (SHR), since they represent a widely used model of essential hypertension (Pintérová et al, 2011). SHR strain was obtained during the 70 's by Okamoto et al, 1963, who started breeding Wistar-Kyoto rats with high blood pressure. Hypertension occurs at about 5-6 weeks of age. Systolic pressure reaches in adulthood values between 180 and $200 \mathrm{mmHg}$ (Zicha and Kuneš, 1999). Starting between 40 and 50 weeks of age, SHR develop cardiovascular diseases (Conrad et al, 1995). SHR rats at 3 months have a hypertrophy of the left ventricle, at 2 years a heart failure. We can find in SHR proteinuria and reduced clearance. The predicted causes of hypertension in the SHR are kidney disorders (Churchill et al, 2002). Wistar-Kyoto rats (WKY) are genetically identical to the SHR and are used as control animals to SHR. The SHR differ from WKY in the major histocompatibility complex and in specific antigens of blood groups.

We tried to detect whether the Wistar-Kyoto and spontaneously hypertensive rats differ in vascular response of a. femoralis to norepinephrine-induced contraction and acetylcholine-induced relaxation. A. femoralis represents large conductance arteries, which undergo remodeling and exhibit endothelial dysfunction during hypertension (Líšková et al, 2010). 
We compared the difference in a. femoralis reactivity to norepinephrine and acetylcholine in adult (5 month old) and aged (12 month old) WKY rats and SHR.

Norepinephrine, as the mediator of the sympathetic nervous system, is involved in regulating blood pressure and causes vascular smooth muscle contraction by elevation of cytosolic free $\mathrm{Ca}^{2+}$. Much of the calcium that activates the contractile apparatus enters the cells through L-type voltage-dependent calcium channels (LVDCC) (Sanders, 2001). The calcium influx through L-VDCC is necessary for norepinephrine-induced contraction of arteries (Paulis et al, 2007). An elevated level of norepinephrine is present in essential hypertension and contributes to endothelial dysfunction (Jeffrey et al, 1998). Endothelial function reflects the relaxation of arteries to acetylcholine. Acetylcholine-induced relaxation is generally used to assess endothelial function. For these reasons, the ability of a. femoralis to relax after cumulative concentrations of acetylcholine was evaluated.

\section{Materials and methods}

Experiments were carried out in adult and aged male WistarKyoto and spontaneously hypertensive rats. The animals were divided into four groups: 5 month old Wistar-Kyoto rats (WKY 5), 12 month old Wistar-Kyoto rats (WKY 12), 5 month old spontaneously hypertensive rats (SHR 5) and 12 month old spontaneously hypertensive rats (SHR 12). All animals were housed under standard laboratory conditions (temperature $23 \pm 1{ }^{\circ} \mathrm{C}, 12$-hour lightdark cycle, standard pelleted diet for laboratory rats, tap water ad libitum). All experimental procedures and protocols were approved by the Ethical Committee of the Institute of Physiology, Academy of Sciences of the Czech Republic, conform to European Convention on Animal Protection and Guidelines on Research Animal Use.

Animals were anesthetized with ether and blood pressure was measured directly by the puncture of carotid artery. After human sacrifice of animals, we isolated their femoral arteries and cut them into 2-4 $\mathrm{mm}$ long segments. They were subsequently placed in Mulvany-Halpern isometric myograph (M 510A, DMT, Denmark). As the nutrient solution, a modified Krebs-Henseleit solution was used (KHS, $119 \mathrm{mM} \mathrm{NaCl}, 4.7 \mathrm{mM} \mathrm{KCl}, 1.17 \mathrm{mM} \mathrm{MgSO}_{4}, 25 \mathrm{mM}$ $\mathrm{NaHCO}_{3}, 1.18 \mathrm{mM} \mathrm{KH}_{2} \mathrm{PO}_{4}, 0.03 \mathrm{mM}$ EDTA, $2.5 \mathrm{mM} \mathrm{CaCl}_{2}, 200$ $\mathrm{mg} / 1$ ascorbic acid, $2 \mathrm{~g} / 1$ glucose), which was heated to $37^{\circ} \mathrm{C}$ and bubbled with $\mathrm{O}_{2}$. After 30 minutes of blood vessels stabilization, we added progressively increasing doses of norepinephrine $\left(3 \times 10^{-8} \mathrm{M}, 10^{-7} \mathrm{M}, 3 \times 10^{-7} \mathrm{M}, 10^{-6} \mathrm{M}, 3 \times 10^{-6} \mathrm{M}, 10^{-5} \mathrm{M}, 3 \times 10^{-5} \mathrm{M}\right.$, $\left.10^{-4} \mathrm{M}\right)$, following by increasing doses of acetylcholine $\left(3 \times 10^{-8} \mathrm{M}\right.$, $\left.10^{-7} \mathrm{M}, 3 \times 10^{-7} \mathrm{M}, 10^{-6} \mathrm{M}, 3 \times 10^{-6} \mathrm{M}\right)$.

The results were evaluated according the amplitude of contractions to norepinephrine of vascular segment records from myograph. They were expressed as vessel wall tension in $\mathrm{mN}$ / $/ \mathrm{mm}$. The relaxation of the segments was evaluated against the maximum amplitude of contraction to the highest dose of norepinephrine and was expressed in percentage.

The data were expressed as the mean \pm SEM. The statistical significance of differences was evaluated by the Student's t-test and a significance threshold was defined as $\mathrm{p}<0.05$.

\section{Results}

Responses to cumulative concentrations of norepinephrine: The age-dependent comparison of norepinephrine-induced contractions has not shown any significant difference between adult and aged animals. In both strains used in our experiments, there was a tendency to increased norepinephrine-induced contraction in arteries of aged animals (Fig. 1). The vessels of SHR expressed a higher norepinephrine-induced contraction compared to vessels of WKY (Fig. 1). The results showed a stronger ability of femoral artery to contract after administration of norepinephrine in the model of experimental hypertension.

Responses to cumulative concentrations of acetylcholine: Acetylcholine-induced relaxation of femoral arteries of hypertensive or aged rats was not changed compared to control vessels (Fig. 2). 5 month old WKY compared to 5 month old SHR rats indicated a slightly better relaxation properties, which reached the only significant value at the highest concentration of acetylcholine $(-81.51 \pm 1.54 \%$ WKY vs $-66.11 \pm 1.82 \%$ SHR $) .12$

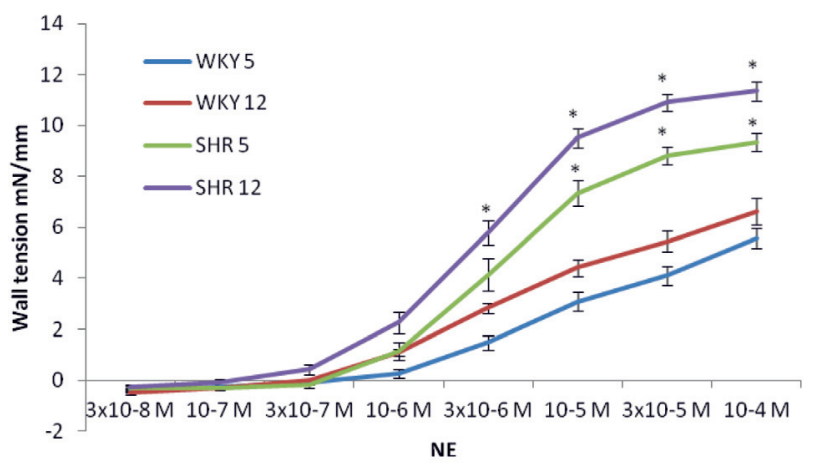

Fig. 1. Reactivity of a. femoralis - comparison of contractions to cumulative concentrations of norepinephrine from normotensive and hypertensive rats. WKY 5 - 5 month old Wistar-Kyoto rats, WKY 12 - 12 month old Wistar-Kyoto rats, SHR $5-5$ month old spontaneously hypertensive rats, SHR 12 - 12 month old spontaneously hypertensive rats. $\mathrm{NE}$ - norepinephrine. ${ }^{*} \mathrm{p}<0.05$.

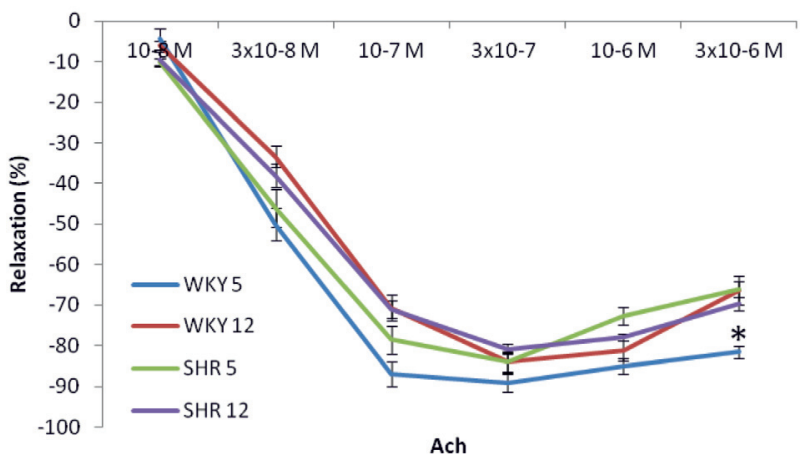

Fig. 2. Reactivity of precontracted a. femoralis - comparison of relaxation responses to cumulative concentrations of acetylcholine from normotensive and hypertensive rats. WKY 5 - 5 month old Wistar-Kyoto rats, WKY 12-12 month old Wistar-Kyoto rats, SHR 5 - 5 month old spontaneously hypertensive rats, SHR 12-12 month old spontaneously hypertensive rats. Ach - acetylcholine. * $\mathrm{p}<\mathbf{0 . 0 5}$. 
month old SHR and 12 month old WKY showed no differences in acetylcholine-induced relaxation.

\section{Discussion}

Endothelium is involved in vascular homeostasis through excretion of relaxing and constricting factors. Decreased availability of NO, increased production of thromboxane $\mathrm{A}_{2}, \mathrm{PGH}_{2}$ and superoxide anion are associated with endothelial dysfunction present in hypertension. These changes lead to the reduction of endothelium-dependent vasodilation and/or to the increase in responses to vasoconstrictor stimuli (Davel et al, 2011).

The principal findings of our study can be summarized as follows. NE-induced contractions of femoral arteries were augmented in hypertensive rats compared to Wistar-Kyoto rats. The NE-induced contractions from the same strain of adult and aged rats did not show significant differences, but showed a tendency to stronger contractions with a higher age. Acetylcholine-induced relaxation was not impaired neither with age, nor with the presence of hypertension.

Blood pressure results from the balance of vasoconstrictors (mainly sympathetic nervous system) and vasodilators (mainly nitric oxide and endothelium-derived hyperpolarizing factor). Nitric oxide and norepinephrine are antagonists in the control of calcium influx through L-type voltage-dependent calcium channels. Spontaneously hypertensive rats are characterized by an absolute enhancement of sympathetic nervous system (Pintérová et al, 2009). High blood pressure resulting from sympathetic hyperactivity and vasodilator deficiency was documented in hereditary hypertriglyceridemic rats (Kuneš et al, 2002), spontaneously hypertensive rats (Paulis et al, 2007; Hojná et al, 2007; Kuneš et al, 2008) or Dahl rats (Zicha et al, 2001; Dobešová et al, 2002). Experimental models represented by spontaneously hypertensive rats and by ouabaine-induced hypertension had disturbed relaxation and constriction responses to various agents compared to normotensive animals (Xavier et al, 2009; Wenceslao et al, 2011). It has been shown that age affects endothelial function. Increasing age reduces the endothelium-dependent relaxation in rat muscle arterioles (Muller-Delp et al, 2002). This effect was also observed in other vascular beds (Egashira et al, 1993, Gerhard et al, 1996; Mayhan et al, 1990; Tominagana et al, 1994). Our experiments did not show a reduced endothelium-dependent relaxation in hypertensive compared to normotensive animals, either due to age of rats or because SHR rats did not suffer from an absolute, but rather from a relative NO deficiency (Pintérová et al, 2009).

The reason for the increased contractile ability of blood vessels to norepinephrine might be functional changes in sympathetic nervous system and in the renin-angiotensin-aldosterone system, leading to an enhanced calcium influx through voltage-dependent calcium channels (Grassi et al, 1998). Renin-angiotensin-aldosterone system amplifies the effect of the sympathetic nervous system peripherally and centrally as well (Balt et al, 2003).

In conclusions, we predict that an increased norepinephrineinduced contraction occurs even before development of reduced acetylcholine-induced relaxation as a manifestation of endothelial dysfunction in SHR. In our experiment hypertension more influenced endothelial dysfunction than aging.

\section{References}

1. Balt JC, Mathy MJ, Pfaffendorf M, Van Zwieten PA. Sympatho-inhibitory actions of irbesartan in pithed spontaneously hypertensive and Wistar-Kyoto rats. Fundam. Clin Pharmacol 2003; 17: 83-91.

2. Churchill PC, Brooks WW, Hayes JA, Sen S, Robinson KG, Bing OH. Increased genetic susceptibility to renal damage in the stroke-prone spontaneously hypertensive rat. Kidney Int 2002; 61 (5): 1794-800.

3. Conrad CH, Brooks WW, Hayes JA, Sen S, Robinson KG, Bing OH. Myocardial fibrosis and stiffness with hypertrophy and heart failure in the spontaneously hypertensive rat. Circulation 1995; 91 (1): 161-70.

4. Davel AP, Wenceslau CF, Akamine EH et al. Endothelial dysfunction in cardiovascular and endocrine-metabolic diseases: an update. Braz J Med Biol Res 2011; 44: 920-932.

5. Egashira K, Inou T, Hirooka Y et al. Effects of age on endothelium-dependent vasodilation of resistance coronary artery by acetylcholine in humans. Circulation 1993; 88: 77-81.

6. Félétou M, Vanhoutte PM. Endothelial dysfunction: a multifaceted disorder. Am J Physiol Heart Circ Physiol 2006; 291: H985-H1002.

7. Gerhard M, Roddy MA, Creager SJ, Creager MA. Aging progressively impairs endothelium-dependent vasodilation in forearm resistance vessels of humans. Hypertension 1996; 27: 849-853.

8. Grassi G, Cattaneo BM, Seravalle G, Lanfranchi A, Mancia G. Baroreflex control of sympathetic nerve activity in essential and secondary hypertension. Hypertension 1998; 31: 68-72.

9. Hojná S, Kadlecová M, Dobešová Z, Valoušková V, Zicha J, Kuneš J. The participation of brain NO synthase in blood pressure control of adult spontaneously hypertensive rats. Mol Cell Biochem 2007; 297: 21 v29.

10. Jeffrey G, Dickhout and Robert MK, Lee W. Blood pressure and heart rate development in young spontaneously hypertensive rats. Am J Physiol Heart Circulat Physiol 1998; 274: H794-H800.

11. Kuneš J, Dobešová Z, Zicha J. Altered balance of main vasopressor and vasodepressor systems in rats with genetic hypertension and hypertriglyceridaemia. Clin Sci 2002; 102: 269-277.

12. Kuneš J, Dobešová Z, Musilová A et al. Hemodynamic characterization of recombinant inbred statins: 20 years after. Hypertens Res 2008; 31: 1659-1668.

13. Mayhan WG, Faraci FM, Baumbach GL, Heistad DD. Effects of aging on responses of cerebral arterioles. Am J Physiol Heart Circ Physiol 1990; 258: H1138-H1143.

14. Muller-Delp JM, Spier SA, Ramsey MW, Delp MD. Aging impairs endothelium-dependent vasodilation in rat skeletal muscle arterioles. Am J Physiol Heart Circ Physiol 2002; 283: H1662-H1672.

15. Paulis L, Líšková S, Pintérová M, Dobešová Z, Kuneš J, Zicha J. Nifedipine-sensitive noradrenergic vasoconstriction is enhanced in spontaneously hypertensive rats: the influence of chronic captopril treatment. Acta Physiol (Oxf) 2007; 191: 255-266.

16. Pintérová M, Kuneš J, Zicha J. Altered neural and vascular mechanisms in hypertension. Physiol Res 2011; 60: 381-402.

17. Sanders KM. Invited reiew: mechanisms of calcium handling in smooth muscles. J Appl Physiol 2001; 91: 1438-1449.

18. Tominaga M, Fujii K, Abe I, Takata Y, Kobayashi K, Fujishima M. Hypertension and ageing impair acetylcholine-induced vasodilation in rats. $\mathbf{J}$ Hypertens 1994; 12: 259-268.

19. Wenceslau CF, Davel AP, Xavier FE, Rossoni LV. Long-term ouabain treatment impairs vascular function in resistance arteries. J Vasc Res 2011; 48: 316-326.

20. Xavier FE, Davel AP, Fukuda LE, Rossoni LV. Chronic ouabain treatment exacerbates blood pressure elevation in spontaneously hypertensive rats: the role of vascular mechanisms. J Hypertens 2009; 27: 1233-1242.

21. Zicha J, Kuneš J. Ontogenetic aspects of hypertension development: analysis in the rat. Physiol Rev 1999; 79: 1277-1282.

22. Zicha J, Dobešová Z, Kuneš J. Relative deficiency of nitric oxide-dependent vasodilation in salt hypertensive Dahl rats: the possible role of superoxide anions. J Hypertens 2001; 19: 247-254.

Received June 7, 2012 Accepted July 20, 2013 